\title{
Visualization of the interaction between tris and lysozyme with a biosensor based on total internal reflection imaging ellipsometry
}

\author{
Tengfei Kang a,b,1, Yu Niu ${ }^{\text {a,1 }}$, Gang Jin ${ }^{\text {a,* }}$ \\ a NML, Institution of Mechanics, Chinese Academy of Sciences, 15 Bei-si-huan West Road, Beijing 100190, China \\ b University of the Chinese Academy of Sciences, 19 Yu-quan Road, Beijing 100049, China
}

\section{A R T I C L E I N F O}

Available online 12 March 2014

\section{Keywords:}

Biosensor

Total internal reflection imaging ellipsometry

Weak affinity interaction

Tris

Lysozyme

\begin{abstract}
A B S T R A C T
Weak affinity interactions among biomolecules have attracted more and more attentions for they play a significant role in organisms. However, it is difficult to characterize these interactions due to the limitation of the detection approaches. The biosensor based on total internal reflection imaging ellipsometry (TIRIE) is a real-time analysis method for biomolecular interaction detection with the advantages of high throughput and sensitivity. The interaction between tris and lysozyme (LZM), a typical example of weak affinity interactions, has been detected with TIRIE as a trial. Tris is immobilized on the gold thin film substrate to form the biosensing surface and then LZM as well as its negative controls is delivered to the tris-immobilized surface. The interaction process between tris and LZM is recorded by TIRIE biosensor to form a real-time curve and its dissociation constant is deduced as $7.6 \times 10^{-5} \mathrm{M}$, which is in agreement with the results in the reported work by other investigators. The results indicate that TIRIE biosensor is competent for weak affinity interaction analysis.
\end{abstract}

C 2014 Elsevier B.V. All rights reserved.

\section{Introduction}

Biomolecular interactions in organism are of great importance for a number of necessary functions in biological bodies [1-4]. Although varying significantly from one to another, the biomolecular interactions can be divided into two categories [5-7]: strong affinity interactions and weak affinity interactions. The interactions, the apparent dissociation constant $\left(K_{D}\right)$ of which is lower than $10^{-6} \mathrm{M}$ [5], are considered as the strong affinity interactions, for example the specific binding between antigens and their immunoglobulin $G$ type antibodies; while the weak affinity interactions are defined as $\mathrm{K}_{\mathrm{D}}$ more than $10^{-6} \mathrm{M}$ [5], such as the pathway of immune response [8], molecular recognition [9], cell adhesion [10] and microorganism infections [11]. Weak affinity interactions are mainly formed by hydrogen bonds, van del Waals interactions and their binding complexes are usually transient and unstable $[5,12]$, which gives rise to the great difficulty to characterize.

The biosensor based on total internal reflection imaging ellipsometry (TIRIE) which is developed from the conventional surface plasmon resonance (SPR) technique with the introduction of imaging ellipsometry method has been utilized as an automatic real-time analysis tool to detect biomolecular interaction processes. TIRIE biosensor is performed under the total internal-reflection mode [13-16] and hence can observe the dynamic process of the biomolecular interaction with a sensitivity 10 times

\footnotetext{
* Corresponding author at: 15 Bei-si-huan West Road, Beijing 100190, China. Tel./fax: + 861082544138 .

E-mail address: gajin@imech.ac.cn (G. Jin).

${ }^{1}$ Authors have equal contribution to this work.
}

larger than the conventional SPR in theory [14]. After constructing the TIRIE engineering model and optimizing its optical setting systematically, TIRIE biosensor has been used for the dynamic analysis of several biomolecular interactions [17] and the real-time detection results can achieve as good sensitivity as expected in theory. These advantages might render TIRIE biosensor the capability of weak affinity interaction detection.

The interaction between tris and lysozyme (LZM) whose $K_{D}$ is deduced to the magnitude of $10^{-5} \mathrm{M}$ by high-performance affinity chromatography [18] has been reported as a typical weak affinity interaction. Tris is a widely-used compound to prepare biomolecule buffer. However, the specific binding between tris and proteins which may cause false-positive results in many experiments [18] is commonly ignored because of the detection difficulty. In this investigation, the interaction between tris and LZM has been detected with TIRIE biosensor to evaluate its capability of weak affinity interaction detection.

\section{Materials and methods}

\subsection{TIRIE biosensor}

The principle of TIRIE biosensor has been described in references [13, 19] and its engineering model in our lab (shown in Fig. 1) [20] is mainly composed of an imaging ellipsometer operated under the total internal reflection mode and a micro-fluidic reactor with the throughput of 24 independent reaction cells. By a prism, the bottom of which tightly contacts with the glass side of a TIRIE biosensor substrate, the ellipsometric imaging system can be coupled with the micro-fluidic reactor and can be used for the dynamic analysis. The ellipsometric imaging system 


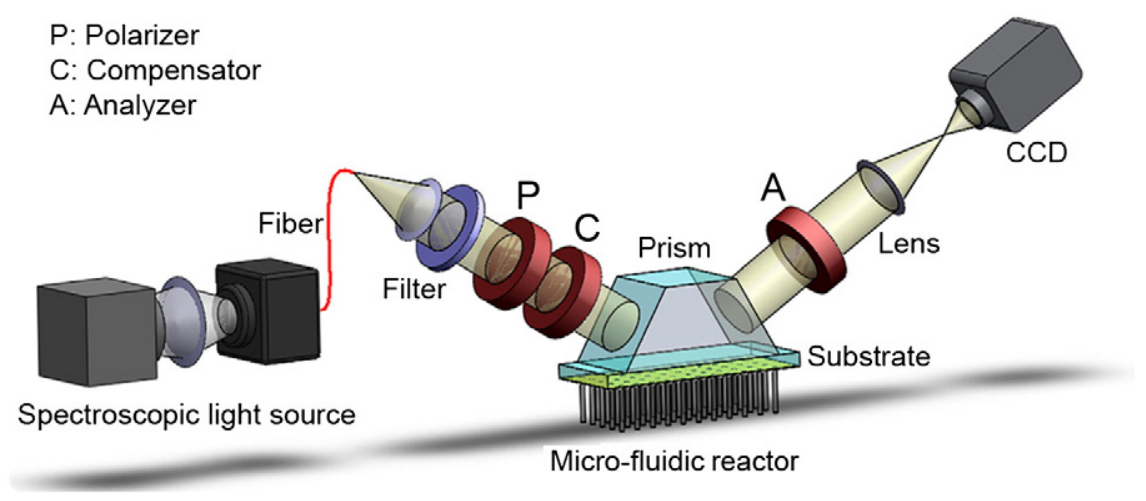

Fig. 1. The schematic diagram of the engineering model of the TIRIE biosensor in our lab [20].

uses a Xe lamp as its light source and a wavelength selector to form an expanded collimating light probe in a certain wavelength. The light beam from the optical fiber goes through a polarizer and a quarter wave plate (compensator) and then penetrates into the sidewall of the coupling prism perpendicularly. Since the incident angle is set larger than the total internal reflection angle, the light beam is reflected totally at the substrate interface. An evanescent wave appearing at the gold/ ambient interface is utilized as the optical probe to detect the mass surface concentration change of biomolecules absorbing on the substrate. With the immobilization of ligand molecule and the recognition to the target molecule, the protein layers vary on the substrate, leading to a change of the surface mass concentration. The polarization of the evanescent wave is modified by the formation of biomolecule layers on the surface so that the polarization state of the reflection light changes at the same time. After the reflection light beam passes through another dichroic polarizer (analyzer) and focusing lens, its intensity is detected by the charge coupled device (CCD) camera in the format of 16-bit grayscale image. Since the time interval to capture and store the reflection light intensity is less than $1 \mathrm{~s}$, the complete process of the biomolecular interaction can be continuously recorded to form a real-time curve (illustrated in Fig. 2). Compared with the conventional SPR biosensor that has been commonly used for protein interaction analysis as a label-free tool, TIRIE biosensor introduces the phase-sensitive ellipsometric method and the imaging sampling approach, obtaining the improvement in detection sensitivity and the capability of high throughput detection.

\subsection{Compounds and substrates}

1-(3-Dimethylaminopropyl)-3-ethylcarbodiimidehydrochloride (EDC), N-hydroxyl-succinimide (NHS),11-mercaptoundecanoic acid (MUA), tris(hydroxymethyl)amino methane, human serum albumin (HSA), ovalbumin (OVA), LZM, phosphate buffered saline buffer (PBS, $\mathrm{pH}=7.4$ ) and 1-iodonaphthalene (its refractive index is near 1.72 ) as the refractive index matching oil are all purchased from Sigma-Aldrich. SF10 glass slides which are coated by $50 \mathrm{~nm}$ thickness gold thin film are produced in our lab as TIRIE biosensor substrates. Hydrogen peroxide, concentrated sulfuric acid and pure ethanol are in analytical grade. Ultra-pure water is obtained from a MILLI-Q purification system $\left(18.2 \mathrm{~m} \Omega\right.$ at $\left.25^{\circ} \mathrm{C}\right)$ and used to prepare all the solutions.

\subsection{TIRIE biosensor substrate preparation}

TIRIE biosensor substrates are cleaned in piranha solution $\left(\mathrm{H}_{2} \mathrm{SO}_{4}\right.$ : $\mathrm{H}_{2} \mathrm{O}_{2}=3: 1, \mathrm{v} / \mathrm{v}$ ) for $30 \mathrm{~min}$ and then washed with ultra-pure water and pure ethanol alternately for three times. After being dried under pure nitrogen flow, the substrates are immersed into MUA ethanolic solution at the concentration of $1 \mathrm{mM}$ for $18 \mathrm{~h}$. Eventually, the
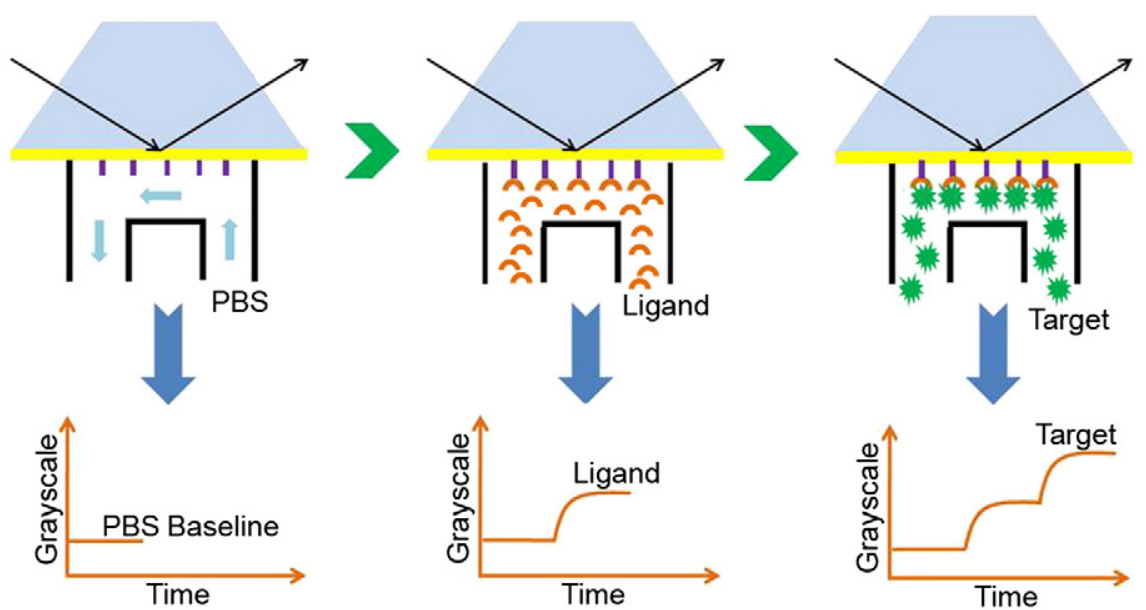

Real-time curve for the interaction between a ligand and its target

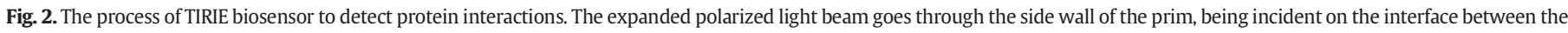

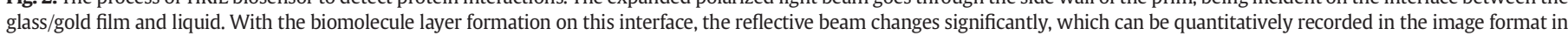
grayscale. By processing a series of continuous images, biomolecule reactions that take place on the gold surface can be converted into a real-time curve. 
modification substrates are rinsed by ethanol and water alternately to remove the excessive MUA and are stored in pure ethanol.

\subsection{Tris and LZM interaction visualization}

Tris immobilization is carried out in the micro-fluidic reactor. $40 \mu \mathrm{L}$ of a mixture solution prepared with NHS and EDC at the concentration of $0.05 \mathrm{~mol} / \mathrm{ml}$ and $0.2 \mathrm{~mol} / \mathrm{ml}$ in deionized water is passed across the gold surface of the substrate at a flow rate of $5 \mu \mathrm{L} / \mathrm{min}$. With NHS and EDC, carboxyl groups can form sulfo-NHS-ester and are able to immobilize tris by reacting to the amino groups of tris. After rinsing with PBS, $75 \mu \mathrm{L}$ of $1 \mathrm{M}$ tris is delivered to each reaction cell at a flow rate of $5 \mu \mathrm{L} / \mathrm{min}$ to form the tris sensing surface. Then, PBS is immediately added to each reaction cell to rinse the tris surface. Once TIRIE biosensor signal keeps stable, $50 \mu \mathrm{L}$ of LZM solutions whose concentration ranges from $3.3 \mu \mathrm{M}$ to $52.8 \mu \mathrm{M}$ is delivered to the tris-immobilized surface at a flow rate of $5 \mu \mathrm{L} / \mathrm{min}$. Eventually, PBS is passed to all the reaction cells to rinse the substrate. The entire process above is continuously recorded in the format of grayscale image by TIRIE biosensor and converted into a real-time curve to present the biosensor signal change.

\section{5. $K_{D}$ deduction of tris and LZM interaction}

The interaction between tris and LZM has been monitored by observing changes in TIRIE biosensor responses. The ratio of tris and lysozyme involved in their interaction is equal to $1: 1$, which is proved by the results of molecular docking method in a reported work by other investigators [18]. Assuming pseudo-first-order interaction kinetics, the net rate of tris and LZM complex formation measured with TIRIE biosensor can be expressed by Langmuir adsorption isotherm as Eq. (1) [21,22].

$d R_{t} / d t=\mathrm{k}_{\mathrm{a}} C\left(\mathrm{R}_{\max }-R_{t}\right)-\mathrm{k}_{\mathrm{d}} R_{t}$

where $d R_{t} / d t$ is the net rate to form the complex of tris and LZM. $\mathrm{k}_{\mathrm{a}}$ and $\mathrm{k}_{\mathrm{d}}$ are the association and disassociation rate constants, respectively. $\mathrm{R}_{\max }$ represents the maximum binding capacity of LZM on the substrate in TIRIE biosensor signal. $R_{t}$ is the TIRIE biosensor signal at a certain time and its value is equivalent to the complex of tris and LZM, so that $\left(R_{\max }\right.$ $-R_{t}$ ) is the number of free binding sites of tris left on the substrate. $C$ is the LZM concentration in the analyte solution.

At equilibrium, $d R_{t} / d t$ is equal to 0 . Thus, Eq. (1) becomes Eq. (2).

$\mathrm{k}_{\mathrm{d}} R_{e q}=\mathrm{k}_{\mathrm{a}} C\left(\mathrm{R}_{\max }-R_{e q}\right)$

where $R_{e q}$ represents the LZM binding amount at equilibrium in TIRIE biosensor signal. Since $K_{D}$ is equal to $k_{d} / k_{a}$, Eq. (2) is converted into Eq. (3).

$1 / R_{e q}=\mathrm{K}_{\mathrm{D}} / \mathrm{R}_{\max } \cdot 1 / \mathrm{C}+1 / \mathrm{R}_{\max }$

A plot of $1 / R_{e q}$ against $1 / C$ can be used to obtain $K_{D}$ which is considered as the ratio of the slope and intercept of the fitting curve.

\section{Results and discussions}

\subsection{Characterization of tris immobilization}

Ellipsometric parameters and contact angle measurements are utilized to characterize tris immobilization on MUA modification gold surface. All the ellipsometry experiments are performed at the incidence angle of $70^{\circ}$ with a $632.8 \mathrm{~nm}$ He-Ne laser by a conventional ellipsometer which is produced by SENTECH Instruments $\mathrm{GmbH}$. Compared with the bare gold, the ellipsometric parameter delta of the MUA modification surface decreases about $1.4^{\circ}$, indicating that a layer forms on the gold surface. Contact angle measurements are carried out by a home-made optical equipment. After incubation in MUA solution, the contact angle of the gold surface goes significantly down about $40^{\circ}$. The surface wettability change is in agreement to the expectation of MUA immobilization on the gold surface.

Since tris molecular weight is as low as $121 \mathrm{Da}$, it is difficult to characterize its adsorption directly. In that case, OVA is introduced as a control to prove tris immobilization on the MUA modification surface. The molecular weight of OVA is similar to LZM but OVA does not react with tris. OVA solution prepared under the same condition to LZM is delivered to the tris immobilized surface and MUA surface, respectively. By comparing the TIRIE biosensor signal change on the both surfaces, it can be deduced whether tris is immobilized on the MUA modification surface. The corresponding real-time results are shown in Fig. 3. On the MUA modification surface, TIRIE biosensor signal goes up about 800 grayscale after OVA addition. The increase which cannot be completely removed by PBS rinsing must have been contributed to the covalent immobilization of OVA on the MUA modification gold surface. On the contrary, TIRIE biosensor signal on tris immobilized surface increases less than 200 grayscale before and after injecting OVA solution, which is much less than that on tris surface. Tris is difficult to occupy the entire MUA modification surface after the immobilization process, so MUA left on the surface might be likely to react to OVA covalently, causing the slight increase of TIRIE biosensor signal. The obvious difference between tris and MUA surface can prove that tris is immobilized on the MUA surface.

\subsection{Tris and LZM interaction detection}

In order to confirm the specificity of the interaction between tris and LZM, OVA and HSA that have similar molecular weight to LZM are introduced as the negative controls. After tris immobilization, LZM, HSA and OVA solutions at the same concentration are injected independently to react with tris surface. Their results are shown in Fig. 4. With OVA and HSA, only slight increase less than 100 grayscale can be observed in TIRIE biosensor signal. Since OVA and HSA cannot react with tris, the change in TIRIE biosensor signal should be attributed to the nonspecific adsorption on the modified gold surface. However, LZM leads to about 300 rise in grayscale value which is remarkably more than that of OVA and HSA, indicating that LZM can recognize tris specifically. Furthermore, the increase caused by the specific interaction completely disappears after rinsing with PBS solution, suggesting that the binding

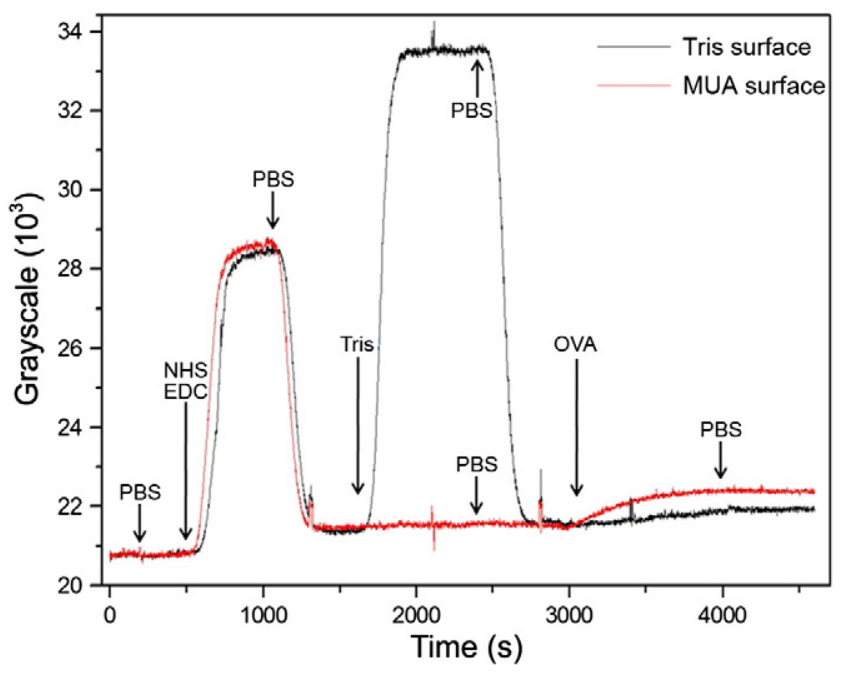

Fig. 3. The real-time curve to confirm tris immobilization. Tris solution at the concentration of $1 \mathrm{M}$ and PBS as the control are delivered to the MUA modification gold surface, respectively. Then, OVA solution at the same concentration of $0.2 \mathrm{mg} / \mathrm{mL}$ is added to tris immobilized and MUA surfaces. 


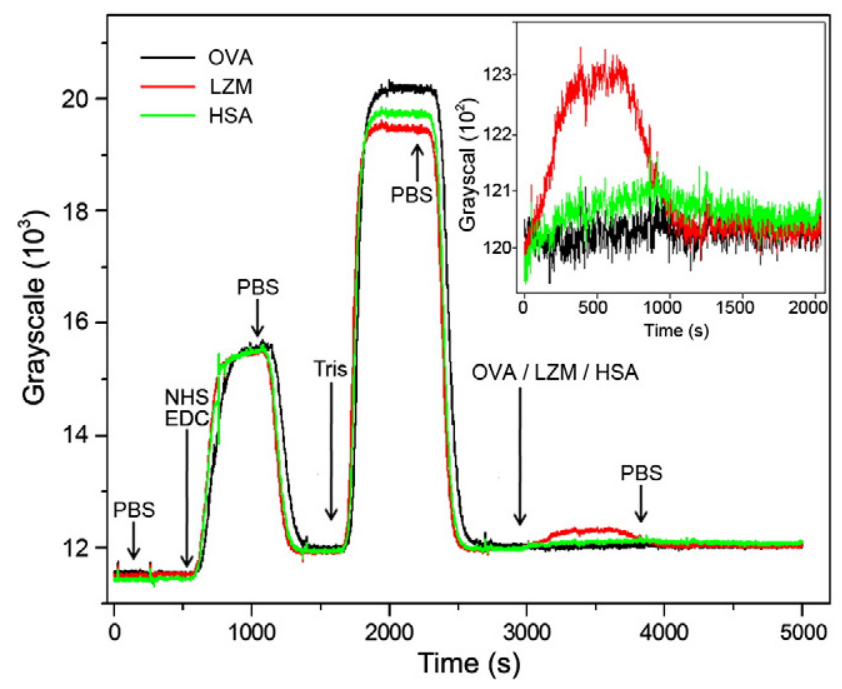

Fig. 4. The real-time curve to confirm the specific interaction between tris and LZM. After tris immobilization on the surface, LZM and its negative controls OVA and HSA at the concentration of $6.6 \mu \mathrm{mol} / \mathrm{L}$ are added to the tris immobilized surface. The inset in the figure represents the recognition process between tris and LZM as well as its negative controls.

complex formed by tris and LZM interaction is unstable and easily to decompose.

\section{3. $K_{D}$ deduction of tris and LZM interaction}

To measure $K_{D}$ value of the interaction between tris and LZM, LZM solution at different concentrations is delivered to tris immobilized surface and the result is presented in Fig. 5. With the increase of LZM concentration, the TIRIE biosensor signal goes up steadily after the addition of LZM solution. In terms of Eq. (3) in Section 2.5, the inverse of TIRIE biosensor signal in grayscale is plotted against the reciprocal of LZM concentration to deduce $K_{\mathrm{D}}$ of the interaction between tris and LZM (shown in Fig. 6). $\mathrm{K}_{\mathrm{D}}$ of the tris and LZM interaction is calculated as $7.6 \times 10^{-5} \mathrm{M}$, which is consistent to the results obtained in the previous work by other investigators [18].

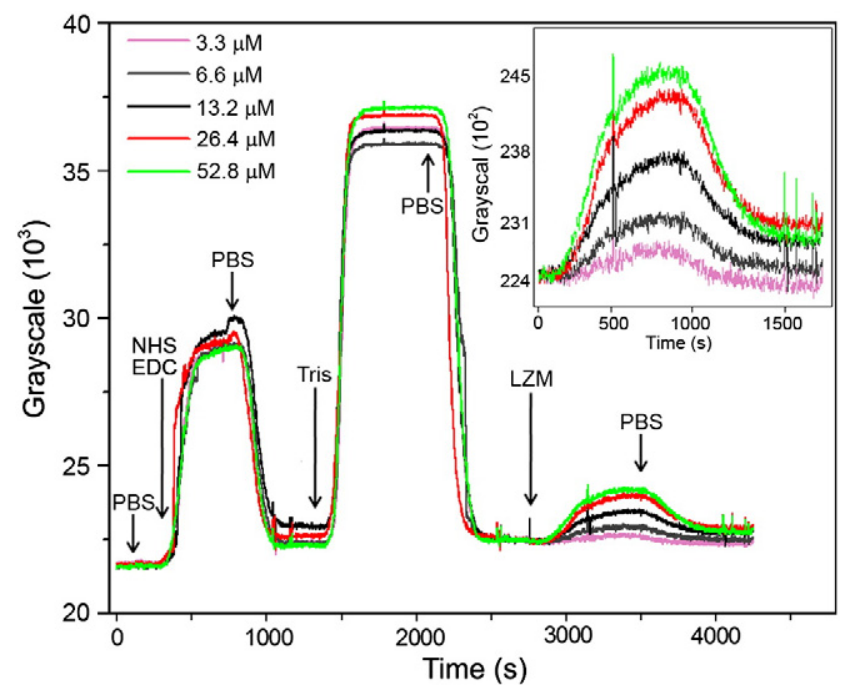

Fig. 5. The real-time curve for the interaction between tris and LZM at different concentrations. LZM is diluted to the concentrations of 3.3, 6.6, 13.2, 26.4 and $52.8 \mu \mathrm{mol} / \mathrm{L}$ and then delivered to the tris immobilized surface. The inset in the figure is clearly to show the specific binding process between tris and LZM.

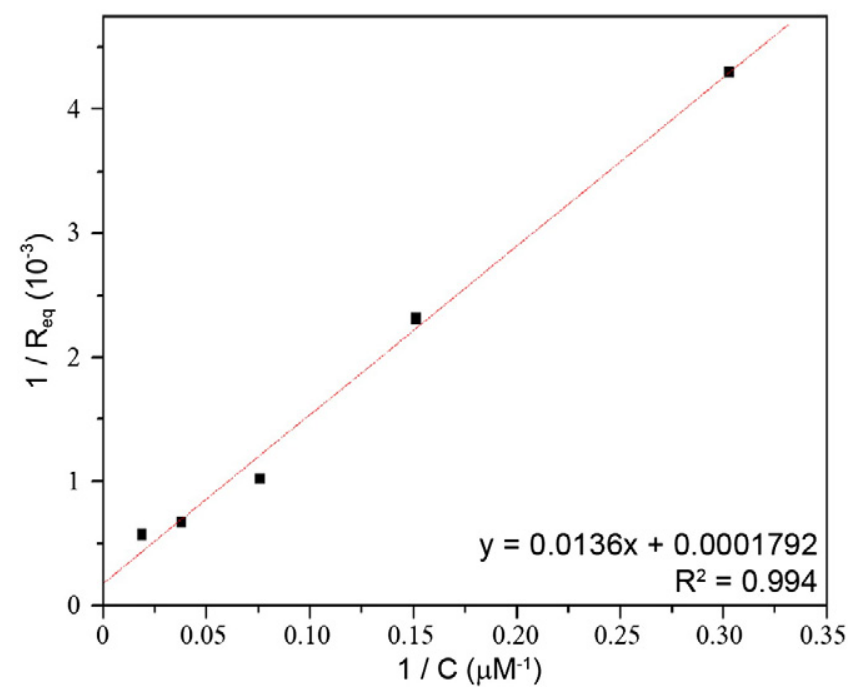

Fig. 6. The fitting curve to deduce $K_{D}$ of the tris and LZM interaction. In terms of Eq. (3), $1 / R_{e q}$ as the $y$ axis is plotted against $1 / C$ as the $x$ axis and then processed by linear fitting. $K_{D}$ is considered as the ratio of the slope and intercept of the fitting curve.

\section{Conclusions}

The dynamic process of the interaction between tris and LZM has been visualized by the TIRIE biosensor as a trial to detect weak affinity interactions. By the introduction of OVA and HSA as the negative controls, the specific binding between tris and LZM is obviously observed in the real-time curve obtained with TIRIE biosensor. $\mathrm{K}_{\mathrm{D}}$ of tris and LZM interaction is in good agreement to other methods described in the reported work by other investigators [18]. It is indicated that TIRIE biosensor is competent as a tool to study weak affinity interactions.

\section{Acknowledgment}

The authors sincerely acknowledge Dr. Ana S. Viana, Dr. Jorge P. Correia, Dr. Ana C. Mourato, Ms. Inês Almeida, and Mr. Joaquim T. Marques in the University of Lisbon for giving help in gold surface modification and Mr. Wei Liu in the University of Chinese Academy of Sciences for language improvement.

The authors acknowledge the financial support to the National Natural Science Foundation of China (21305147), to the International Science \& Technology Cooperation Program of China (2012DFG31880), to the Seventh Sino-Portugal Scientific and Technological Cooperation of 2013-2015, to the National Basic Research Program of China (2009CB320302), and to the National High Technology Research Development Program of China (2008AA02Z419).

\section{References}

[1] D. Nelson, M. Cox, Lehninger Principles of Biochemistry, 5th ed. W. H. Freeman, New York, 2008

[2] A. Finkelstein, O. Ptitsyn, Protein Physics, 1st ed. Academic Press, London, 2008.

[3] A. Nel, L. Madler, D. Velegol, T. Xia, E. Hoek, P. Somasundaran, F. Klaessig, V. Castranova, M. Thompson, Nat. Mater. 8 (2009) 543.

[4] R. Jelinek, Cellular and Biomolecular Recognition, 1st edition Wiley-VCH, Weinheim 2009.

[5] M. Strandh, Insights into Weak Affinity Antibody-antigen Interactions, University of Kalmar, Kalmar, 2000.

[6] S. Ohlson, M. Strandh, H. Nilshans, J. Mol. Recognit. 10 (1997) 135.

[7] M. Strandh, B. Persson, H. Roos, S. Ohlson, J. Mol. Recognit. 11 (1998) 188.

[8] D. Zehn, S.Y. Lee, M.J. Bevan, Nature 458 (2009) 211.

[9] H. Kumar, T. Kawai, S. Akira, Int. Rev. Immunol. 30 (2011) 16.

[10] Q. Shen, Y. Wang, E. Kokovay, G. Lin, S.M. Chuang, S.K. Goderie, B. Roysam, S. Temple, Cell Stem Cell 3 (2008) 289.

[11] M. Benoit, B. Desnues, J.L. Mege, J. Immunol. 181 (2008) 3733.

[12] M.D. Duong-Thi, E. Meiby, M. Bergstrom, T. Fex, R. Issaksson, S. Ohlson, Anal. Biochem. 414 (2011) 138. 
[13] G. Jin, Y.H. Meng, L. Liu, Y. Niu, S. Chen, Q. Cai, T.J. Jiang, Development of biosensor based on imaging ellipsometry and biomedical applications, Thin Solid Films 519 (2011) 2750.

[14] A.V. Nabok, A. Tsargorodskaya, A.K. Hassan, N.F. Starodub, Appl. Surf. Sci. 246 (2005) 381

[15] G. Jin, Phys. Status Solidi A 205 (2008) 810.

[16] W. Yuan, H.P. Ho, S.Y. Wu, Y.K. Suen, S.K. Kong, Sens. Actuators A 151 (2009) 23.

[17] L. Liu, Y.Y. Chen, Y.H. Meng, S. Chen, G. Jin, Thin Solid Films 519 (2011) 2758.
[18] L. Quan, D.G. Wei, X.L. Jiang, Y. Liu, Z.Y. Li, N. Li, K. Li, F. Liu, L.H. Lai, Anal. Biochem. 378 (2008) 144

[19] L. Liu, Y. Niu, S. Chen, Y.H. Meng, H.W. Ma, G. Jin, Sci. China Ser. G Phys. Mech. Astron. 53 (2010) 1805.

[20] L. Liu, A. Viallat, G. Jin, Sens. Actuators B-Chem. 190 (2014) 221.

[21] X. Liu, J.Y. Wei, D.Q. Song, Z.W. Zhang, H.Q. Zhang, G.M. Luo, Anal. Biochem. 314 (2003) 301.

[22] T. Fischer, M. Beyermann, K.W. Koch, Biochem. Biophys. Res. Commun. 285 (2001) 463. 